BULL. AUSTRAL. MATH. SOC.

75007,68610

VOL. $26(1982), 469-470$.

\title{
TIME-FREQUENCY ANALYSIS OF SPEECH \\ VIA MODULATED SPLINES
}

\author{
Mi ChaEL John FLAHERTy
}

The acoustic properties of speech signals have been the subject of intensive research by engineers for over forty years. However, human beings remain far superior to machines in their ability to produce and interpret speech and until comparable performances are attained speech analysis will remain an active area of research.

Speech is produced by a time-varying resonant system (the articulatory mechanism) and is processed by a system capable of highlighting both temporal features and periodicities in acoustic signals (the peripheral auditory mechanism). Analysis techniques which attempt to combine a time domain and frequency domain approach, for the purposes of signal analysis, are generally referred to as time-frequency analysis techniques.

This thesis examines a number of time-frequency analysis techniques within a common framework and examines their limitations. The major limitations are seen to result from the limited ability of the basis functions to adapt to changing signal characteristics and highlight local signal non-stationarities. In an attempt to overcome these limitations a novel means of time-frequency analysis has been developed via a new class of basis functions which will be referred to here as 'modulated splines'. The efficient implementation of the method and an investigation of its properties was a major goal of the study. It is shown that these basis functions lead to a mathematically tractable closed form approximation of the speech signal. The method is not based solely upon a production model

Received 12 August 1982. Thesis submitted to University of Queenslan Queensland, August 1981. Degree approved June 1982. Supervisors: Dr L. Skattebol and $\mathrm{Dr} J$. Belward. 
viewpoint nor does it assume signal stationarity but rather attempts to highlight aspects of the speech signal which are of perceptual importance.

An efficient method for obtaining a representation of speech relative to the 'modulated spline' basis functions is presented. Experimental work was carried out to examine the properties of 'modulated splines' in representing stationary voiced speech. Conventional methods of spectral estimation generally rely on Fourier techniques. The 'modulated spline' method of time-frequency analysis is shown to rely on spline smoothed estimates of broadband instantaneous frequency. The properties considered were the accurate 'modulated spline' reconstructions of time domain and frequency domain (in a Fourier sense) information and in particular the relationships between the 'modulated spline' representation and the widely accepted formant parameter description of speech. For a detailed quantitative evaluation the stationary vowels were obtained from a digital formant synthesizer incorporating a realistic glottal source. Efficient 'modulated spline' to formant parameter transformations are presented which compare favourably with existing formant parameter extraction methods.

Stop consonant-vowel pairs of real speech are used as a test set for 'modulated spline' analysis because of their non-stationarities which are most prevalent within the interval of the burst. Waveform comparisons and informal listening tests have demonstrated the high quality of approximation possible for 'modulated spline' reconstructions.

The 'modulated spline' representation is shown to be efficiently obtained by first decomposing the speech signal into a sum of analytic signal components. For the speech samples used in this product, this was achievable by broad bandpass filtering. However, there are situations where this strategy can present problems. The major source of problems is seen to arise in situations where more than one dominant analytic signal component is present within a single broadband. An analytical investigation of this problem is undertaken and an alternate method of 'dominant' analytic signal separation is proposed.

The thesis concludes with a summary of major results and recommendations for further work.

62 Thynne Avenue, Norman Park, Queensland 4170, Australia. 